\title{
ANÁLISE DA EROSIVIDADE DAS CHUVAS ASSOCIADA AOS PADRÕES DE PRECIPITAÇÃO PLUVIAL NA REGIÃO DE RIBEIRÃO DAS LAJES (RJ) ${ }^{(1)}$
}

\author{
Roriz Luciano Machado ${ }^{(2)}$, Daniel Fonseca de Carvalho( ${ }^{(3)}$, Janaina \\ Ribeiro Costa ${ }^{(4)}$, Dionízio Honório de Oliveira Neto ${ }^{(5)}$ \& Marinaldo \\ Ferreira Pinto ${ }^{(6)}$
}

\begin{abstract}
RESUMO
As características físicas das chuvas determinam sua erosividade, que constitui importante fator nas relações de causa e efeito do processo erosivo dos solos, sendo sua caracterização fundamental para o planejamento conservacionista. Este trabalho foi realizado com o objetivo de avaliar a distribuição dos atributos das chuvas (altura precipitada, duração, energia cinética, intensidade máxima em 30 min) e índices de erosividade $\left(\mathrm{EI}_{30}\right.$ e $\left.\mathrm{KE}>25\right)$ em relação aos meses do ano e padrões de precipitação pluvial, para uma série de dados pluviográficos referentes à região de Ribeirão das Lajes (RJ). Os dados foram analisados em delineamento inteiramente casualisado em esquema fatorial do tipo 12 (meses) x 3 (padrões), e também, por meio da análise multivariada de componentes principais (ACP). Com os resultados obtidos, foi possível constatar que: o $\mathrm{EI}_{30}$ anual de 6.772,04 $\mathrm{MJ} \mathrm{mm} \mathrm{ha}^{-1} \mathrm{~h}^{-1}$ está concentrado de novembro a março, representando $81,3 \%$ do total. De acordo com a técnica de ACP, as características das chuvas e índices de erosividade que estiveram mais correlacionadas com os meses e padrões de precipitação pluvial, foram: energia cinética, $\mathrm{EI}_{30}, \mathrm{KE}>25$, altura precipitada e $I_{30}$. Os padrões de precipitação pluvial avançado e atrasado predominam na época mais chuvosa e na mais seca, respectivamente; e a ACP permitiu separar de forma adequada as características das chuvas e erosividade ao longo do ano e padrões de precipitação pluvial para Ribeirão das Lajes.
\end{abstract}

Termos de indexação: erosão hídrica, $\mathrm{EI}_{30}, \mathrm{KE}>25$.

\footnotetext{
${ }^{(1)}$ Recebido para publicação em outubro de 2007 e aprovado em agosto de 2008.

(2) Doutorando em Agronomia, Ciência do Solo, Universidade Federal Rural do Rio de Janeiro - UFRRJ. BR 465, km 7, CEP 23890 000 Seropédica (RJ). Bolsista CAPES. E-mail: roriz@ufrrj.br

(3) Professor do Departamento de Engenharia, UFRRJ. Bolsista do CNPq. E-mail: carvalho@ufrrj.br

(4) Pesquisadora Embrapa Agrobiologia. BR 465, km 7, CEP 23890-000 Seropédica (RJ). E-mail: janaina@cnpab.embrapa.br

(5) Mestrando em Agronomia-Fitotecnia, UFRRJ. E-mail: dionisioneto@yahoo.com.br

(6) Estudante de Engenharia Agrícola, UFRRJ. Bolsista de Iniciação Científica FAPERJ. E-mail: mfpufrrj@yahoo.com.br
} 


\title{
SUMMARY: ANALYSIS OF RAINFALL EROSIVITY ASSOCIATED TO PLUVIAL PRECIPITATION PATTERNS IN THE RIBEIRÃO DAS LAJES REGION, RIO DE JANEIRO STATE, BRAZIL
}

\begin{abstract}
The rainfall characteristics determine erosivity, which is an important factor in the cause - effect relationship in soil erosion. The characterization of rainfall is therefore of fundamental importance for conservation planning. The present study aimed to evaluate the distribution of rainfall characteristics (depth, duration, kinetic energy, maximum intensity in $30 \mathrm{~min})$ and erosivity indexes $\left(E I_{30}\right.$ and $\left.\mathrm{KE}>25\right)$ in relation to the months of the year and to precipitation patterns for a series of pluviographic data in the region of Ribeirão das Lajes, Rio de Janeiro state, Brazil. The data were analysed by univariate variance analysis in a completely randomized design in a 12 (months) $\times 3$ (precipitation patterns) factorial scheme, as well as by principal component multivariate analysis (PCA). Results led to the conclusion that: $81.3 \%$ of the total annual $E I_{30}$ of 6,772.04 $\mathrm{MJ} \mathrm{mm} \mathrm{ha}^{-1}$ $h^{-1}$ was concentrated in the period from November to March. In agreement with the ACP technique, the rainfall characteristics and erosivity index that most closely correlated with the months and precipitation patters were kinetic energy, $E_{30}, K E>25$, depth, and maximum intensity in $30 \mathrm{~min}$. The patterns of early and delayed rainfall predominated in the moist and in the dry periods, respectively; The PCA showed a clear separation of rainfall characteristics and erosivity along the year, and rainfall patterns for the region of Ribeirão das Lajes.
\end{abstract}

Index terms: water erosion, $E I_{30}, K E>25$.

\section{INTRODUÇÃO}

Abrangendo os Estados de São Paulo, Minas Gerais e Rio de Janeiro, a bacia do rio Paraíba do Sul é uma das regiões do país mais influenciadas pela erosão hídrica, onde mais de $20 \%$ de sua área, correspondendo a mais de um milhão de hectares, encontra-se em terras de alta ou muito alta vulnerabilidade à erosão. A despeito de sua importância no abastecimento de água para mais de 9 milhões de pessoas da região metropolitana do Rio de Janeiro - RMRJ (CEIVAP, 2002), a região do médio vale Paraíba é considerada a mais crítica de toda a bacia por concentrar a maior produção de sedimentos.

A erosão acelerada é considerada como uma das formas mais prejudiciais de degradação dos solos tropicais (Cogo et al., 2003). Além de reduzir a fertilidade e, conseqüentemente, a produtividade das culturas por meio da perda de terra, água e nutrientes (Távora et al., 1985), esse fenômeno pode causar sérios problemas ambientais como o assoreamento (Shen \& Lai, 1996) e a contaminação de corpos d'água (Bertol et al., 2004).

Em relação aos fatores relacionados à erosão hídrica, Silva et al. (1999) citam em sua revisão o clima (quantidade, intensidade e duração das chuvas), classe e características do solo (físicas, químicas e mineralógicas), relevo (declividade, comprimento de rampa e forma do terreno), umidade antecedente, interceptação pela cobertura vegetal, tipo de cultivo (sistema de preparo) e uso de práticas conservacionistas, como os mais importantes no processo. A maioria desses fatores é utilizada em modelos empíricos de erosão, como é o caso da Equação Universal de Perda de Solo (USLE), em que quantificando-se os parâmetros do modelo no local de estudo, é possível prever as perdas anuais de terra, em razão do sistema de cultivo e práticas de manejo adotadas, e assim, orientar o planejamento conservacionista (Cogo et al., 2003; Alburquerque et al., 2005).

Dos fatores responsáveis no processo, a capacidade da chuva em causar erosão, ou seja, sua erosividade, é considerada como o fator mais ativo da erosão hídrica (Moura \& Medeiros, 1987). Tem seu potencial erosivo quantificado a partir das características físicas da precipitação pluvial e expressa por índices de erosividade como o $\mathrm{EI}_{30}$ e $\mathrm{KE}>25$, os mais utilizados (Montebeller et al., 2007). Além de possibilitar a obtenção do fator $\mathrm{R}$ (índice de erosividade anual) da USLE, o conhecimento da distribuição da erosividade $\left(\mathrm{EI}_{30}\right)$ ao longo do ano permite alertar sobre os períodos mais críticos visando à adoção de estratégias para a redução da perda de solo e nutrientes (Távora et al., 1985).

As correlações entre a erosividade das chuvas e a perda de solo no Brasil nem sempre são altas, variando de 0,57 a 0,77 (Silva et al., 1997a). Normalmente, uma chuva de maior erosividade causa uma perda de solo elevada, mas, em alguns casos, o mesmo nível de perda pode ser obtido após uma chuva de erosividade menor. Segundo Wischmeier (1959), a obtenção da melhor correlação entre erosividade e perdas de solo é dificultada pela falta de conhecimento das características físicas das chuvas. Embora o potencial erosivo seja bem estudado em todas as regiões 
brasileiras (Oliveira Júnior \& Medina, 1990; Silva et al., 1997b; Moreti et al., 2003; Montebeller, 2005; Albuquerque et al., 2005), ainda são poucos os estudos desenvolvidos sobre características físicas de chuvas como às relacionadas com a variação de sua intensidade ao longo da duração, ou seja, com os padrões de precipitação.

Horner \& Jens (1941) padronizaram as chuvas em três diferentes padrões, de acordo com o tempo de ocorrência do pico de intensidade em relação ao tempo total da chuva, como padrão avançado (AV), intermediário (IN) e atrasado (AT). No padrão avançado, o pico de intensidade da chuva ocorre no primeiro terço de sua duração total, o intermediário no terço médio, e o tipo atrasado no terço final da chuva. Conforme Flanagan et al. (1988), nos padrões intermediário e atrasado são esperadas as maiores perdas de solo tendo em vista maior umidade antecedente, pois em solo úmido a capacidade de infiltração é menor e a desagregação é favorecida pelo impacto das gotas de chuvas, causando selamento superficial e, consequentemente, escoamento superficial.

Segundo Evangelista et al. (2005), o conhecimento das características físicas das chuvas com relação aos padrões de precipitação, permite realizar com maior exatidão estudos com chuva simulada, por utilizar condições mais próximas às condições reais, ou seja, das chuvas naturais. De acordo com Eltz et al. (2001), a maioria dos estudos com chuvas simuladas utiliza um único padrão de chuva, o padrão constante, o que não é coerente em regiões tropicais onde as perdas de solo são mais correlacionadas às chuvas de alta intensidade e curta duração. Esses autores quantificaram as perdas de solo e água submetida aos padrões avançado, intermediário, atrasado e constante, utilizando chuva simulada, e concluíram que as taxas de perda de solo foram de 37; 49 e $91 \%$ maiores no padrão atrasado que no avançado, intermediário e constante, respectivamente.

Carvalho et al. (2008), estudando o efeito das características da chuva natural em parcelas experimentais de perda de solo no município de Seropédica (RJ), ocupadas com diferentes práticas de manejo, concluíram que os padrões AV, IN e AT ocorreram em 64,$6 ; 21,3$ e $14,1 \%$ dos eventos de precipitação avaliados, sendo os mesmos responsáveis por 35,$1 ; 6,6$ e $58,3 \%$ das perdas de solo em um Argissolo Vermelho-Amarelo.

Em função dos muitos fatores que influenciam o processo erosivo com relação à chuva, o uso de uma análise de componentes principais, que apresenta como objetivos analisar quais variáveis explicam a maior parte da variabilidade total e reduzir o número de variáveis (Bouroche \& Saporta, 1982), pode constituir uma ferramenta importante para caracterizar épocas do ano e padrões de precipitação quanto às variáveis relacionadas aos eventos de chuva e, conseqüentemente, sua erosividade. Nesse sentido, este trabalho teve como objetivo avaliar os atributos altura precipitado, duração, intensidade máxima em $30 \mathrm{~min}\left(\mathrm{I}_{30}\right)$, energia cinética (Ec) e os índices de erosividade $\left(\mathrm{EI}_{30} \mathrm{e} \mathrm{KE}>25\right)$ para os diferentes meses do ano e padrões de precipitação pluvial, na região de Ribeirão das Lajes, utilizando estatística uni e multivariada.

\section{MATERIAL E MÉTODOS}

Este trabalho foi desenvolvido com dados pluviográficos da estação Lajes (código 02243251), fornecidos pela LIGHT (Light Serviços de Eletricidade SA) e relativos ao período de 1966 a 1983. A estação se localiza no município de Piraí (RJ), nas coordenadas $22^{\circ} 42^{\prime} 38^{\prime \prime}$ de latitude Sul e $43^{\circ} 52^{\prime} 41^{\prime \prime}$ de longitude Oeste e apresenta altitude de $462 \mathrm{~m}$ (Gonçalves et al., 2006). Foram utilizados os registros de pluviogramas diários, com amplitude de $10 \mathrm{~mm}$ de altura precipitada e menor escala de leitura de $0,1 \mathrm{~mm}$ e tempo de registro de $24 \mathrm{~h}$ com menor escala de leitura de $10 \mathrm{~min}$.

Inicialmente, os dados pluviográficos foram convertidos para o formato digital mediante a digitalização com uso do Sistema para Digitalização de Pluviogramas (HidroGraph 1.02), desenvolvido pelo Grupo de Pesquisa em Recursos Hídricos da Universidade Federal de Viçosa (Gonçalves, 2002). Os critérios adotados para a individualização das chuvas erosivas foram os mesmos propostos por Wischmeier $\&$ Smith (1965) e modificados por Cabeda (1976), ou seja, uma chuva foi considerada independente quando estava separada de outra por no mínimo $6 \mathrm{~h}$ com precipitação pluvial inferior a $1 \mathrm{~mm}$, e considerada erosiva quando a altura precipitada era superior a $10 \mathrm{~mm}$, ou quando era igual ou superior a $6 \mathrm{~mm}$, em um período máximo de $15 \mathrm{~min}$.

De posse do arquivo digital, foi utilizado o programa computacional CHUVEROS (Cogo et al., 2003) para o cálculo dos índices de erosividade $\mathrm{EI}_{30} \mathrm{e} \mathrm{KE}>25$ para toda a série de dados existente.

O experimento foi analisado utilizando o delineamento inteiramente casualizado em esquema fatorial $12 \times 3$, correspondendo aos 12 meses do ano e aos três padrões de precipitação (AV, IN e AT), por meio de uma análise de variância (ANAVA). Portanto, os dados de chuva foram organizados em padrões de precipitação em cada mês de ocorrência do ano, os quais foram assumidos como tratamentos. As variáveis avaliadas foram os índices $\mathrm{EI}_{30} \mathrm{e} \mathrm{KE}>25$, além das demais características das chuvas disponibilizadas pelo CHUVEROS: altura precipitada, duração das chuvas, energia cinética e $\mathrm{I}_{30}$.

Visando verificar a adequação dos dados às pressuposições da análise paramétrica, foi verificada a normalidade e homogeneidade de variâncias dos resíduos do modelo por meio do software SAEG 4.0, e em seguida, foi realizada a análise de variância e o 
teste de comparação de médias de Scott Knott (5\%), utilizando o software SISVAR (Ferreira, 2003). Para todas as variáveis analisadas, houve a necessidade de transformação dos dados em $\log (X+1)$ por não atenderem as pressuposições da análise paramétrica. Também foi realizada a análise multivariada, denominada Análise de Componentes Principais (ACP), para a série histórica de dados pluviográficos de Ribeirão das Lajes (RJ), considerando como amostras os fatores meses do ano e os padrões de precipitação. As variáveis analisadas no programa Canoco (Ter Braak \& Smilauer, 1998), por meio do método multivariado ACP, foram as mesmas usadas na univariada (ANAVA).

\section{RESULTADOS E DISCUSSÃO}

Com exceção dos meses de junho e julho, observase que o padrão de chuva mais freqüente na região é o avançado, principalmente nos meses que compõem a estação chuvosa (novembro a março) (Quadro 1). Nesse período, o padrão avançado apresenta, em média, freqüência igual ou maior a $50 \%$ com relação aos demais padrões. Na média anual, o percentual de chuvas associado a cada padrão foi de 45,7, 23,6 e $30,8 \%$, para os padrões AV, IN e AT, respectivamente.

Em estudos realizados em Santa Maria (Mehl et al., 2001) e Quaraí (Peñalva Bazano et al., 2007), no Rio Grande do Sul, foram encontrados resultados relativamente semelhantes aos obtidos em Ribeirão das Lajes (RJ). Por outro lado, para a região de Seropédica (RJ), Carvalho et al. (2005) encontraram os percentuais de 61,$3 ; 23,6$ e 15,1\%, respectivamente, para os mesmos padrões, os quais foram mais próximos aos encontrados para Lavras, MG (Evangelista et al., 2005). Comparando os resultados, percebe-se que apesar da proximidade das duas localidades (Seropédica e Piraí), o regime pluviométrico apresentase bem diferenciado em virtude principalmente das características associadas ao relevo, que, por sua vez, influenciam na distribuição e ocorrência dos eventos erosivos. Embora o padrão avançado seja o mais freqüente no período de novembro a março, o seu efeito esperado pode ser menos agravante com relação à perda de solo, tendo em vista ser o padrão onde ocorre menor desestruturação dos agregados, menor selamento superficial, e conseqüentemente, menor escoamento superficial e carreamento de partículas (Eltz et al., 2001). Vale ressaltar que as práticas agrícolas na região que geralmente deixam o solo desprotegido são realizadas neste período, fazendo com que um planejamento conservacionista deva sempre ser adotado visando minimizar as perdas de solo e nutrientes.

Com relação à média mensal de precipitação, é possível observar a ocorrência de dois períodos distintos, sendo um de maior precipitação (agosto abril) e outro que corresponde à época seca (maio julho). Em relação à média dos padrões de precipitação, observa-se que as maiores alturas precipitadas ocorrem sob padrão avançado $(28,51 \mathrm{~mm})$, seguidas do intermediário (21,64 $\mathrm{mm}$ ) e atrasado (16,40 $\mathrm{mm})$. $\mathrm{O}$ período do ano que mais contribuiu para a diferença entre os padrões corresponde ao período de outubro a dezembro e o mês de abril. Em outubro e novembro, em média, as maiores alturas precipitadas estão no padrão avançado, enquanto que em dezembro e abril, essas ocorreram tanto no padrão avançado quanto no intermediário.

Considerando que maiores perdas de solo são esperadas em decorrência de chuvas classificadas como atrasadas e intermediárias, é possível constatar que para o padrão intermediário não há diferença estatística da altura precipitada ao longo dos meses do ano. No padrão atrasado, as maiores alturas precipitadas ocorrem em média no período de agosto a março, com exceção de dezembro. No padrão avançado, as maiores alturas precipitadas ocorrem de setembro a abril, ou seja, na época das águas.

Em relação à média mensal de duração das chuvas ao longo do ano, não houve diferença estatística entre os meses. Quanto à duração na média dos padrões de precipitação, observa-se que eventos com maiores durações ocorrem nos padrões avançado e intermediário. Analisando-se os meses do ano que mais influenciaram nas diferenças de duração das chuvas entre os padrões de precipitação, é possível constatar que os meses de outubro, dezembro e janeiro foram os que apresentaram maiores valores, os quais

Quadro 1. Distribuição percentual e número de eventos de chuva ocorridos para cada padrão de precipitação, no período de 1966 a 1983 na estação de Ribeirão das Lajes (RJ)

\begin{tabular}{|c|c|c|c|c|c|c|c|c|c|c|c|c|c|}
\hline Padrão & Jan & Fev & Mar & Abr & Mai o & Jun & Jul & Ago & Set & Out & Nov & Dez & $\overline{\mathbf{X}}$ \\
\hline $\mathrm{AV}$ & 67,6 & 50,0 & 50,0 & 33,3 & 50,0 & 29,6 & 26,3 & 40,0 & 53,1 & 42,9 & 52,3 & 52,8 & 45,7 \\
\hline IN & 18,6 & 27,5 & 31,3 & 33,3 & 25,0 & 22,2 & 26,3 & 20,0 & 12,5 & 28,6 & 19,8 & 17,6 & 23,6 \\
\hline $\mathrm{AT}$ & 13,7 & 22,5 & 18,8 & 33,3 & 25,0 & 48,1 & 47,4 & 40,0 & 34,4 & 28,6 & 27,9 & 29,6 & 30,8 \\
\hline Total eventos & 102 & 40 & 64 & 48 & 20 & 27 & 19 & 20 & 32 & 63 & 86 & 108 & \\
\hline
\end{tabular}

AV, IN e AT correspondem aos padrões avançado, intermediário e atrasado, respectivamente. 
Quadro 2. Altura precipitada e duração das chuvas para os diferentes padrões de precipitação pluvial ao longo do ano em Ribeirão das Lajes (RJ)

\begin{tabular}{|c|c|c|c|c|}
\hline \multirow{2}{*}{ Mês/média padrão } & \multicolumn{3}{|c|}{ Padrão de precipitação } & \multirow{2}{*}{ Média do mês } \\
\hline & Avançado & Intermediário & Atrasado & \\
\hline & \multicolumn{4}{|c|}{ Altura precipitada (mm) } \\
\hline Janeiro & 32,68 aA & 19,32 aA & 20,21 aA & 28,48 a \\
\hline Fevereiro & 33,65 aA & 29,91 aA & 19,33 aA & 29,40 a \\
\hline Março & $31,31 \mathrm{aA}$ & 31,80 aA & 22,08 aA & 29,73 a \\
\hline Abril & 25,94 aA & 25,81 aA & $13,13 \mathrm{bB}$ & 21,62 a \\
\hline Maio & $18,30 \mathrm{aB}$ & 13,20 aA & $8,00 \mathrm{aB}$ & $14,45 \mathrm{~b}$ \\
\hline Junho & $12,38 \mathrm{aB}$ & 13,33 aA & 11,77 aB & $12,30 \mathrm{~b}$ \\
\hline Jul ho & $16,20 \mathrm{aB}$ & 19,80 aA & $9,22 \mathrm{aB}$ & $13,84 \mathrm{~b}$ \\
\hline Agosto & $17,20 \mathrm{aB}$ & 22,00 aA & 26,57 aA & $21,20 \mathrm{a}$ \\
\hline Setembro & 23,44 aA & 10,75 aA & 14,70 aA & $19,13 \mathrm{a}$ \\
\hline Outubro & 29,52 aA & $13,22 \mathrm{bA}$ & $16,33 \mathrm{bA}$ & 21,10 a \\
\hline Novembro & 28,29 aA & $18,05 \mathrm{bA}$ & $19,23 \mathrm{bA}$ & 23,71 a \\
\hline Dezembro & $29,21 \mathrm{aA}$ & 24,11 aA & $15,03 \mathrm{bB}$ & 24,11 a \\
\hline Média dos padrões & $28,51 \mathrm{~A}$ & $21,64 \mathrm{~B}$ & $16,40 \mathrm{C}$ & 23,71 \\
\hline \multirow[t]{2}{*}{ CV (\%) } & \multicolumn{4}{|c|}{91,19} \\
\hline & \multicolumn{4}{|c|}{ Duração da chuva (h) } \\
\hline Janeiro & 10,17 aA & $7,90 \quad$ aA & $4,33 \mathrm{bA}$ & $8,95 \mathrm{a}$ \\
\hline Fevereiro & $6,92 \mathrm{aA}$ & 10,52 aA & 5,77 aA & 7,65 a \\
\hline Março & $10,20 \mathrm{aA}$ & 9,58 aA & 5,84 aA & 9,19 a \\
\hline Abril & 9,82 aA & 10,48 aA & 6,33 aA & 8,87 a \\
\hline Maio & $5,30 \mathrm{aA}$ & 6,93 aA & 5,32 aA & 5,71 a \\
\hline Junho & 7,54 aA & 8,41 aA & 7,39 aA & 7,66 a \\
\hline Julho & 8,15 aA & 9,78 aA & 7,50 aA & 8,27 a \\
\hline Agosto & 6,93 aA & 9,09 aA & 12,59 aA & 9,23 a \\
\hline Setembro & $12,64 \mathrm{aA}$ & 8,09 aA & 9,05 aA & $10,95 \mathrm{a}$ \\
\hline Outubro & $11,40 \mathrm{aA}$ & 10,51 aA & $5,40 \mathrm{bA}$ & 9,43 a \\
\hline Novembro & 10,45 aA & 7,97 aA & 8,03 aA & 9,28 a \\
\hline Dezembro & $9,37 \mathrm{aA}$ & 8,81 aA & 5,32 bA & 8,07 a \\
\hline Média dos padrões & $9,74 \mathrm{~A}$ & $9,15 \mathrm{~A}$ & $6,57 \mathrm{~B}$ & 8,76 \\
\hline $\mathrm{CV}(\%)$ & \multicolumn{4}{|c|}{90,12} \\
\hline
\end{tabular}

Médias seguidas de mesma letra, nas colunas, para cada padrão e média dos meses (letras minúsculas), e nas linhas entre os padrões de precipitação e médias dos padrões (letras maiúsculas), não diferem entre si pelo teste de Scott Knott ( $\mathrm{p}>$,05).

foram superiores nos padrões avançado e intermediário. Nos demais meses, não houve diferença estatística desse atributo entre os padrões de precipitação. Analisando a duração da chuva ao longo do ano em cada padrão de ocorrência, não houve diferença significativa entre os meses. Observa-se ainda (Quadro 2) que os coeficientes de variação da altura precipitada e duração foram elevados, o que pode estar relacionado à variabilidade característica desse fenômeno nas regiões tropicais.

Analisando a distribuição da energia cinética das chuvas, observa-se que na média mensal, no período de agosto a abril, estão concentrados os maiores valores desse índice, semelhante ao observado para a altura precipitada (Quadro 3). Quanto à média dos padrões, semelhante à altura precipitada, no padrão avançado predominam os maiores valores de Ec, seguido do intermediário e atrasado. Os meses de abril, outubro e dezembro foram os que apresentaram diferença estatística entre os padrões. Em abril e dezembro, os maiores valores de Ec ocorreram nos padrões avançado e intermediário, e, no mês de outubro, no padrão avançado. Com relação ao comportamento dos meses em cada padrão, somente no atrasado houve diferenças estatística para Ec. Nesse padrão, no período de outubro a março (com exceção do mês de dezembro), e o mês de agosto, ocorreram os maiores valores de energia cinética.

Com respeito à distribuição do $\mathrm{I}_{30}$ ao longo do ano, é possível constatar que, em média, os maiores índices estão concentrados no período de novembro a abril e em agosto. Avaliando a distribuição do $\mathrm{I}_{30}$ em relação aos padrões, observa-se que os maiores índices médios ocorreram no padrão avançado $\left(22,35 \mathrm{~mm} \mathrm{~h}^{-1}\right)$, seguido dos padrões intermediário $\left(14,84 \mathrm{~mm} \mathrm{~h}^{-1}\right) \mathrm{e}$ atrasado $\left(14,93 \mathrm{~mm} \mathrm{~h}^{-1}\right)$, que não diferiram entre si estatisticamente. Entre os padrões, somente no mês de outubro houve diferença significativa, com o padrão 
Quadro 3. Distribuição da energia cinética (Ec) e do $I_{30}$ ao longo do ano em relação aos padrões de precipitação em Lajes (RJ)

\begin{tabular}{|c|c|c|c|c|}
\hline \multirow{2}{*}{ Mês/média padrão } & \multicolumn{3}{|c|}{ Padrões de precipitação } & \multirow{2}{*}{ Média dos meses } \\
\hline & Avançado & Intermediário & Atrasado & \\
\hline & \multicolumn{4}{|c|}{ Ec $\left(\mathrm{MJ} m \mathrm{~m} \mathrm{ha}^{-1}\right)$} \\
\hline Janeiro & $7,18 \mathrm{aA}$ & $3,90 \mathrm{aA}$ & $4,71 \mathrm{aA}$ & $6,23 \mathrm{a}$ \\
\hline Fevereiro & $7,78 \mathrm{aA}$ & $6,02 \mathrm{aA}$ & $3,97 \mathrm{aA}$ & $6,44 \mathrm{a}$ \\
\hline Março & $6,89 \mathrm{aA}$ & $6,60 \mathrm{aA}$ & $4,98 \mathrm{aA}$ & $6,44 \mathrm{a}$ \\
\hline Abril & $5,29 \mathrm{aA}$ & $5,27 \mathrm{aA}$ & $2,66 \mathrm{bB}$ & $4,40 \mathrm{a}$ \\
\hline Maio & $4,01 \mathrm{aA}$ & $2,38 \mathrm{aA}$ & $1,48 \mathrm{aB}$ & $2,97 \mathrm{~b}$ \\
\hline Junho & $2,53 \mathrm{aA}$ & $2,52 \mathrm{aA}$ & $2,10 \mathrm{aB}$ & $2,32 \mathrm{~b}$ \\
\hline Julho & $3,22 \mathrm{aA}$ & $3,62 \mathrm{aA}$ & $1,52 \mathrm{aB}$ & $2,52 \mathrm{~b}$ \\
\hline Ag osto & $3,54 \mathrm{aA}$ & $4,13 \mathrm{aA}$ & $5,10 \mathrm{aA}$ & $4,18 \mathrm{a}$ \\
\hline Setembro & $4,52 \mathrm{aA}$ & $1,65 \mathrm{aA}$ & $2,69 \mathrm{aB}$ & $3,59 \mathrm{a}$ \\
\hline Outubro & $6,30 \mathrm{aA}$ & $2,22 \mathrm{bA}$ & $3,29 \mathrm{bA}$ & $4,27 \mathrm{a}$ \\
\hline Novembro & $5,84 \mathrm{aA}$ & $3,62 \mathrm{aA}$ & $4,05 \mathrm{aA}$ & $4,89 \mathrm{a}$ \\
\hline Dezembro & $6,21 \mathrm{aA}$ & $4,86 \mathrm{aA}$ & $2,95 \mathrm{bB}$ & $5,01 \mathrm{a}$ \\
\hline Média dos padrões & $6,11 \mathrm{~A}$ & $4,29 \mathrm{~B}$ & $3,34 \mathrm{C}$ & 4,95 \\
\hline $\mathrm{CV}(\%)$ & \multicolumn{4}{|c|}{$\begin{array}{c}103,92 \\
30\left(\mathrm{~mm} \mathrm{~h}^{-1}\right)\end{array}$} \\
\hline Janeiro & $24,48 \mathrm{aA}$ & $15,75 \mathrm{aA}$ & $27,38 \mathrm{aA}$ & $23,25 \mathrm{a}$ \\
\hline Fevereiro & $31,06 \mathrm{aA}$ & $18,59 \mathrm{aA}$ & $18,98 \mathrm{aA}$ & 24,91 a \\
\hline Março & $24,45 \mathrm{aA}$ & $19,80 \mathrm{aA}$ & $20,88 \mathrm{aA}$ & 22,32 a \\
\hline Abril & $19,09 \mathrm{aA}$ & $16,82 \mathrm{aA}$ & $12,70 \mathrm{aB}$ & $16,20 \mathrm{a}$ \\
\hline Maio & $20,37 \mathrm{aA}$ & $8,46 \mathrm{aA}$ & $7,54 \mathrm{aB}$ & $14,19 \mathrm{~b}$ \\
\hline Junho & $12,04 \mathrm{aA}$ & $8,63 \mathrm{aA}$ & $8,83 \mathrm{aB}$ & $9,74 \mathrm{~b}$ \\
\hline Julho & $14,36 \mathrm{aA}$ & $13,26 \mathrm{aA}$ & $6,36 \mathrm{aB}$ & $10,28 \mathrm{~b}$ \\
\hline Agosto & $15,48 \mathrm{aA}$ & $15,50 \mathrm{aA}$ & $15,94 \mathrm{aA}$ & $15,64 \mathrm{a}$ \\
\hline Setembro & $15,93 \mathrm{aA}$ & $4,82 \mathrm{aB}$ & $11,26 \mathrm{aB}$ & $13,08 \mathrm{~b}$ \\
\hline Outubro & $21,87 \mathrm{aA}$ & $7,49 \mathrm{bB}$ & $14,38 \mathrm{aB}$ & $15,62 \mathrm{~b}$ \\
\hline Novembro & $21,71 \mathrm{aA}$ & $15,81 \mathrm{aA}$ & $17,81 \mathrm{aB}$ & $19,41 \mathrm{a}$ \\
\hline Dezembro & $22,94 \mathrm{aA}$ & $16,95 \mathrm{aA}$ & $12,53 \mathrm{aB}$ & 18,80 a \\
\hline Média dos padrões & $22,35 \mathrm{~A}$ & $14,84 \mathrm{~B}$ & $14,93 \mathrm{~B}$ & 18,65 \\
\hline CV $(\%)$ & \multicolumn{4}{|c|}{89,79} \\
\hline
\end{tabular}

Médias seguidas de mesma letra, nas colunas, para cada padrão e média dos meses (letras minúsculas), e nas linhas entre os padrões de precipitação e médias dos padrões (letras maiúsculas), não diferem entre si pelo teste de Scott Knott ( $p>$,05).

intermediário apresentando, em média, menor $\mathrm{I}_{30}$ que os demais padrões. Dentro de cada padrão, observase que não houve diferença estatística para $\mathrm{I}_{30}$ entre os meses do ano e para o padrão avançado. No entanto, para o padrão intermediário, os menores valores de $\mathrm{I}_{30}$ foram verificados nos meses de setembro e outubro, enquanto que no padrão atrasado, os maiores valores foram constatados de janeiro a março e em agosto.

Semelhante aos atributos anteriores, os coeficientes de variação para a energia cinética e $\mathrm{I}_{30}$ foram muito altos, o que pode está relacionado à grande variabilidade da intensidade da chuva ao longo do ano, atributo utilizado no cálculo da Ec (Foster et al., 1981). Isso pode ter contribuído para a ausência de diferenças não significativas entre as médias dos meses e padrões de precipitação para esses atributos, apesar de terem se mostrado com elevada discrepância.

A figura 1 apresenta a distribuição mensal dos índices de erosividade $\mathrm{EI}_{30}$ e $\mathrm{KE}>25$ em cada padrão de precipitação. É possível observar que ambos os índices apresentam o mesmo comportamento, estando concentrados no período de outubro a março, com predominância do padrão avançado. Para esse padrão, são observados os maiores valores de $\mathrm{EI}_{30}$ e $\mathrm{KE}>25$, com destaque para o mês de janeiro com 1.432,8 MJ mm ha ${ }^{-1} \mathrm{~h}^{-1}$ (Figura 1a) e 17,9 MJ ha-1 (Figura 1b), respectivamente.

Em complemento à figura 1, o quadro 4 apresenta a caracterização dos valores extremos individuais do $\mathrm{EI}_{30}$, em cada padrão de prescipitação e a distribuição anual da erosividade. Seguindo a tendência apresentada na figura 1, é possível verificar que os valores de maior erosividade ocorreram nos meses de novembro a março, sendo que o maior valor foi observado em janeiro (precisamente em 22 de janeiro 1967), quando foi precipitada uma altura de $220 \mathrm{~mm}$ em aproximadamente $4 \mathrm{~h}$, proporcionando uma erosividade de 5.228,0 MJ mm ha ${ }^{-1} \mathrm{~h}^{-1}$. De acordo com Montebeller (2005), que estudou a variabilidade espacial do potencial erosivo das chuvas no Estado do Rio de janeiro, valor desta magnitude é esperado, em média, para o período de um ano na região sul fluminense. 
Conforme LIGHT (1997), dentre os eventos registrados, esse é considerado o de maior magnitude da história da região, que provocou vários deslizamentos de encostas e inundação das usinas hidrelétricas de Nilo Peçanha e Fontes Nova (RJ). Em relação à média mensal de erosividade, desconsiderando os padrões de chuvas, como na maioria dos trabalhos, o $\mathrm{EI}_{30}$ anual é de $6.772,0 \mathrm{MJ} \mathrm{mm} \mathrm{ha}^{-1} \mathrm{~h}^{-1}$, sendo os meses de novembro a março o período de maior erosividade (81,3 \% do total anual). No Estado do Rio de Janeiro, esses valores foram superiores aos encon-
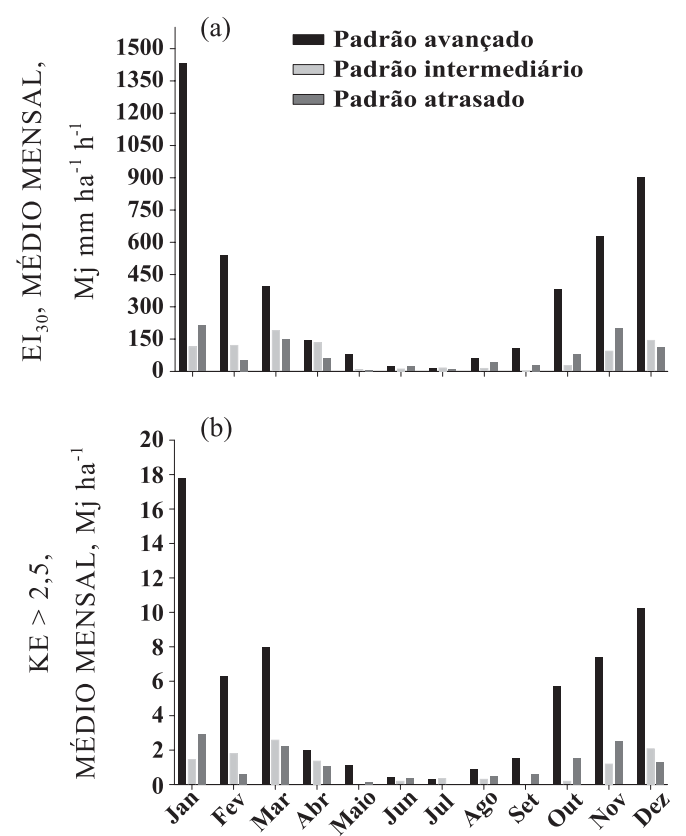

Figura 1. Distribuição mensal do $\mathrm{EI}_{30}$ (a) e $\mathrm{KE}>25$ (b) em Ribeirão das Lajes (RJ), em função dos padrões de precipitação. trados para as cidades de Seropédica e Nova Friburgo, que apresentaram médias mensais de 5.472 e de $5.431 \mathrm{MJ} \mathrm{mm} \mathrm{ha}^{-1} \mathrm{~h}^{-1}$, respectivamente (Carvalho et al., 2005). Comparando com outras regiões do país, esses dados foram inferiores à erosividade de São Manuel-SP, com 7.487 MJ mm ha-1 $\mathrm{h}^{-1}$ (Moreti et al., 2003), de Goiânia, com 8.353,0 $\mathrm{MJ} \mathrm{mm} \mathrm{ha}^{-1} \mathrm{~h}^{-1}$ (Silva et al., 1997b), de Quaraí-RS, com 9.292 MJ mm $\mathrm{ha}^{-1} \mathrm{~h}^{-1}$ (Peñalva Bazano et al., 2007) de Manaus, com 14.129 MJ mm ha-1 $\mathrm{h}^{-1}$ (Oliveira Júnior \& Medina, 1990), e superior a Sumé-PB, com 4.928 MJ mm $\mathrm{ha}^{-1} \mathrm{~h}^{-1}$ (Albuquerque et al., 2005).

Carvalho (1994) classificou a erosividade (R) quanto à magnitude do índice $\mathrm{EI}_{30} \mathrm{em}$ : baixa, se $\mathrm{R}<2.452$; média, se $2.452<\mathrm{R}<4.905$; média a forte, se $4.905<\mathrm{R}<7.357$; forte, se $7.357<\mathrm{R}<9.810$; e muito forte, para $R>9.810$. Com base nessa classificação, a erosividade de Ribeirão das Lajes é considerada de média a forte e, por isso, práticas agrícolas que promovem a movimentação do solo, alterando as condições de superfície do terreno, devem ser utilizadas com atenção, principalmente no período de novembro a março.

Observa-se que o período de novembro a abril, e o mês de agosto apresentaram valores de $\mathrm{EI}_{30}$ estatisticamente significativos, semelhante ao resultado observado para a intensidade máxima de precipitação em 30 min $\left(\mathrm{I}_{30}\right)$ (Quadro 5). Com relação aos padrões de precipitação, os maiores índices médios de $\mathrm{EI}_{30}$ estão associados ao padrão avançado $\left(232,00 \mathrm{MJ} \mathrm{mmha}^{-1} \mathrm{~h}^{-1}\right)$, seguido do intermediário $\left(93,43 \mathrm{MJ} \mathrm{mm} \mathrm{ha}^{-1} \mathrm{~h}^{-1}\right)$ e atrasado $(86,76 \mathrm{MJ} \mathrm{mm}$ $\mathrm{ha}^{-1} \mathrm{~h}^{-1}$ ), semelhante ao observado para os atributos altura precipitada e energia cinética. Analisando os meses que mais contribuíram para a diferença entre os padrões, constata-se que nos meses de dezembro e Abril, os padrões avançado e intermediário não diferiram entre si, e foram superiores ao padrão atrasado. No mês de outubro, o padrão avançado foi

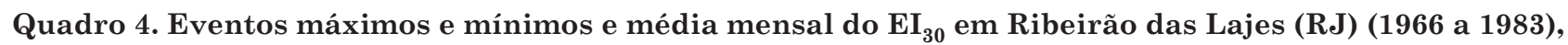
para os diferentes padrões de precipitação

\begin{tabular}{|c|c|c|c|c|c|c|c|}
\hline \multirow{2}{*}{ Mês } & \multicolumn{2}{|c|}{ Padrão avançado } & \multicolumn{2}{|c|}{ Padrão intermediário } & \multicolumn{2}{|c|}{ Padrão atrasado } & \multirow{2}{*}{$\begin{array}{l}\text { Média } \\
\text { mensal }\end{array}$} \\
\hline & Máximo & Mínimo & Máximo & Mínimo & Máximo & Mínimo & \\
\hline & \multicolumn{7}{|c|}{ MJ mm ha ${ }^{-1} h^{-1}$} \\
\hline Janeiro & $5.228,0$ & 0 & 741,7 & 0 & $1.069,5$ & 0,1 & $1.759,33$ \\
\hline Fevereiro & $3.599,7$ & 1,0 & 549,9 & 10,4 & 313,3 & 13,4 & 712,03 \\
\hline Março & $2.912,3$ & 1,2 & 638,8 & 11,0 & $1.197,6$ & 0,2 & 958,46 \\
\hline Abril & 629,3 & 0 & 929,4 & 10,9 & 335,5 & 0,3 & 337,19 \\
\hline Maio & 726,3 & 2,3 & 30,8 & 8,4 & 65,4 & 0,9 & 94,27 \\
\hline Junho & 91,5 & 1,5 & 84,7 & 1,5 & 73,0 & 0,2 & 54,28 \\
\hline Julho & 70,5 & 15,6 & 114,1 & 9,1 & 32,3 & 0,7 & 38,63 \\
\hline Agosto & 509,4 & 2,4 & 126,9 & 16,1 & 276,5 & 24,1 & 117,12 \\
\hline Setembro & 289,0 & 2,5 & 14,8 & 4,0 & 169,0 & 0,9 & 139,99 \\
\hline Outubro & $1.039,4$ & 0,1 & 144,0 & 0,4 & 312,9 & 0,1 & 485,11 \\
\hline Novembro & $2.099,2$ & 0,9 & 243,3 & 0 & 1. 233,3 & 0,1 & 920,91 \\
\hline Dezembro & $2.826,7$ & 0,2 & 646,8 & 1,8 & 326,2 & 0 & $1.154,73$ \\
\hline Total & & & & & & & $6.772,04$ \\
\hline
\end{tabular}


superior aos demais que não diferiram entre si. Nos demais meses do ano, não houve diferença significativa entre os padrões.

Avaliando os meses do ano dentro de cada padrão de ocorrência das chuvas, verifica-se que o $\mathrm{EI}_{30}$ nos padrões avançado e intermediário não apresentou diferenças significativas ao longo dos meses do ano. Já para o padrão atrasado, no período de outubro a março (exceto dezembro) e no mês de agosto predominam os maiores valores de $\mathrm{EI}_{30}$.

Em relação à distribuição do $\mathrm{KE}>25$ ao longo do ano, verifica-se que os maiores valores ocorreram nos meses de janeiro a março, o que era esperado pelo fato deste índice ser calculado utilizando-se apenas as precipitações que apresentam intensidade igual ou superior a $25 \mathrm{~mm} \mathrm{~h}^{-1}$, típicas para esta época do ano. Nos padrões de precipitação, observa-se que no avançado predominam os maiores valores, seguido dos demais padrões, que não diferiram entre si, semelhante ao observado para duração das chuvas. Com relação à variação deste índice entre os padrões ao longo do ano (Quadro 5). Verifica-se que nos meses de outubro e janeiro, os maiores valores de KE > 25 estão associados aos padrões avançado e atrasado. Em dezembro, o avançado e intermediário não diferenciaram entre si e foram superiores ao atrasado. Analisando o comportamento do KE > 25 em cada padrão ao longo do ano, é possível verificar que não houve diferença significativa entre os meses. Os coeficientes de variação dos índices de erosividade foram também elevados, da mesma forma como observado para os demais atributos das chuvas.

Uma característica observada em dezembro é que em praticamente todos os atributos avaliados (altura precipitada, Ec, $\mathrm{EI}_{30}$ e $\mathrm{KE}>25$ ) os maiores valores ocorreram tanto no padrão avançado quanto no intermediário. À medida que os picos de intensidade tendem a ocorrer no terço final de duração da chuva, maior é o potencial de perda de solo esperado, indicando que, na região de estudo, esse mês dentre outros, deve

Quadro 5. Distribuição do $\mathrm{EI}_{30}$ e do $\mathrm{KE}>25$ ao longo do ano em relação aos padrões de precipitação em Ribeirão das Lajes (RJ)

\begin{tabular}{|c|c|c|c|c|}
\hline \multirow{2}{*}{ Mês/média padrão } & \multicolumn{3}{|c|}{ Padrão de precipitação } & \multirow{2}{*}{ Média do mês } \\
\hline & Avançado & Intermediário & Atrasado & \\
\hline & \multicolumn{4}{|c|}{$\mathrm{EI}_{30}\left(\mathrm{MJ} \mathrm{mm} \mathrm{ha} \mathrm{h}^{-1} \mathrm{~h}^{-1}\right)$} \\
\hline Janeiro & $311,47 \mathrm{aA}$ & $90,20 \mathrm{aA}$ & 227,46 aA & $258,72 \mathrm{a}$ \\
\hline Fevereiro & $404,30 \mathrm{aA}$ & $164,26 \mathrm{aA}$ & 87,51 aA & 267,01 a \\
\hline Março & $276,71 \mathrm{aA}$ & $166,00 \mathrm{aA}$ & $183,52 \mathrm{aA}$ & $224,64 \mathrm{a}$ \\
\hline Abril & $134,70 \mathrm{aA}$ & 124,76 aA & $56,65 \mathrm{bB}$ & $105,37 \mathrm{a}$ \\
\hline Maio & 121,93 aA & $20,90 \mathrm{aA}$ & $18,06 \mathrm{aB}$ & $70,71 \mathrm{~b}$ \\
\hline Junho & $40,68 \mathrm{aA}$ & 27,05 aA & $25,12 \mathrm{aB}$ & $30,16 \mathrm{~b}$ \\
\hline Julho & $47,46 \mathrm{aA}$ & $47,70 \mathrm{aA}$ & $11,52 \mathrm{aB}$ & $30,50 \mathrm{~b}$ \\
\hline Agosto & $92,40 \mathrm{aA}$ & $66,50 \mathrm{aA}$ & 90,47 aA & 87,84 a \\
\hline Setembro & 99,08 aA & 8,45 aA & $44,46 \mathrm{aB}$ & $65,62 \mathrm{~b}$ \\
\hline Outubro & 210,76 aA & $22,51 \mathrm{bA}$ & $65,61 \mathrm{bA}$ & $115,50 \mathrm{~b}$ \\
\hline Novembro & $209,74 \mathrm{aA}$ & $74,32 \mathrm{aA}$ & 134,70 aA & $160,62 \mathrm{a}$ \\
\hline Dezembro & $236,84 \mathrm{aA}$ & $113,43 \mathrm{aA}$ & $52,06 \mathrm{bB}$ & $160,38 \mathrm{a}$ \\
\hline Média dos padrões & $232,00 \mathrm{~A}$ & $93,43 \mathrm{~B}$ & $86,76 \mathrm{C}$ & 161,50 \\
\hline \multirow[t]{2}{*}{ CV (\%) } & \multicolumn{4}{|c|}{236,44} \\
\hline & \multicolumn{4}{|c|}{$\mathrm{KE}>25\left(\mathrm{MJ} \mathrm{ha}^{-1}\right)$} \\
\hline Janeiro & $3,87 \mathrm{aA}$ & $1,14 \mathrm{bA}$ & 3,14 aA & $3,26 \mathrm{a}$ \\
\hline Fevereiro & $4,72 \mathrm{aA}$ & $2,44 \mathrm{aA}$ & $1,02 \mathrm{aA}$ & $3,26 \mathrm{a}$ \\
\hline Março & $3,74 \mathrm{aA}$ & $1,93 \mathrm{aA}$ & $2,78 \mathrm{aA}$ & $3,00 \mathrm{a}$ \\
\hline Abril & $1,89 \mathrm{aA}$ & $1,28 \mathrm{aA}$ & 0,99 aA & $1,38 \mathrm{~b}$ \\
\hline Maio & $1,71 \mathrm{aA}$ & $0,00 \mathrm{aA}$ & $0,44 \mathrm{aA}$ & $0,96 \mathrm{~b}$ \\
\hline Junho & $0,75 \mathrm{aA}$ & $0,47 \mathrm{aA}$ & $0,41 \mathrm{aA}$ & $0,52 \mathrm{~b}$ \\
\hline Julho & $0,90 \mathrm{aA}$ & $1,02 \mathrm{aA}$ & $0,00 \mathrm{aA}$ & $0,50 \mathrm{~b}$ \\
\hline Agosto & $1,33 \mathrm{aA}$ & $1,50 \mathrm{aA}$ & $1,01 \mathrm{aA}$ & $1,24 \mathrm{~b}$ \\
\hline Setembro & $1,25 \mathrm{aA}$ & $0,00 \mathrm{aA}$ & $0,86 \mathrm{aA}$ & $0,97 \mathrm{~b}$ \\
\hline Outubro & $3,17 \mathrm{aA}$ & $0,16 \mathrm{bA}$ & $1,29 \mathrm{aA}$ & $1,77 \mathrm{~b}$ \\
\hline Novembro & $2,46 \mathrm{aA}$ & $0,94 \mathrm{aA}$ & $1,70 \mathrm{aA}$ & $1,93 \mathrm{~b}$ \\
\hline Dezembro & $2,70 \mathrm{aA}$ & $1,64 \mathrm{aA}$ & $0,62 \mathrm{bA}$ & $1,90 \mathrm{~b}$ \\
\hline Média dos padrões & $2,92 \mathrm{~A}$ & $1,18 \mathrm{~B}$ & $1,23 \mathrm{~B}$ & 2,07 \\
\hline $\mathrm{CV}(\%)$ & \multicolumn{4}{|c|}{199,85} \\
\hline
\end{tabular}

Médias seguidas de mesma letra, nas colunas, para cada padrão e média dos meses (letras minúsculas), e nas linhas entre os padrões de precipitação e médias dos padrões (letras maiúsculas), não diferem entre si pelo teste de Scott Knott ( $\mathrm{p}>0,05)$. 
receber atenção quanto ao uso de práticas de manejo que mantenham uma boa cobertura vegetal, bem como adoção de outras práticas conservacionistas.

Com o objetivo de analisar de forma conjunta os atributos das chuvas erosivas e índices de erosividade em relação aos meses do ano e padrões de precipitação, foi utilizado a análise multivariada de componentes principais para os dados (Quadros 6 e Figura 2). São apresentados os valores dos dois primeiros componentes principais $\left(\mathrm{Y}_{1}\right.$ e $\left.\mathrm{Y}_{2}\right)$ e a ordenação decrescente das amostras para cada um dos componentes. A percentagem de explicação da variância total dos dados pelos três primeiros componentes principais foi de $96,6 \%$, com contribuições de 72,6; 20,3 e 3,7 \% para $\mathrm{Y}_{1}, \mathrm{Y}_{2}$ e $\mathrm{Y}_{3}$, respectivamente.

A figura 2 apresenta a distribuição das variáveis, dos padrões de precipitação e dos meses do ano em relação aos dois componentes principais $\left(\mathrm{Y}_{1} \mathrm{e} \mathrm{Y}_{2}\right)$. O primeiro eixo referente ao $\mathrm{Y}_{1}$, os meses junho, julho e maio, como também o padrão atrasado (T), destacaramse dos demais, tendo em vista a baixa altura pluviométrica associada a eles. É possível perceber também que os meses de agosto, abril, outubro, dezembro e novembro, além do padrão intermediário, apresentaram comportamento distinto do anterior. As variáveis que contribuíram expressivamente para a

Quadro 6. Valores dos dois componentes principais $\left(\mathrm{Y}_{1}\right.$ e $\left.\mathrm{Y}_{2}\right)$ correspondentes aos meses e padrões de precipitação pluvial, ordenação decrescente das amostras para $Y_{1}$ e $Y_{2}$ e porcentagens de variação nos dados, individual e acumulada, explicada pelos dois primeiros componentes principais

\begin{tabular}{lrrrr}
\hline Amostra & $\mathbf{Y}_{\mathbf{1}}$ & Ordem & $\mathbf{Y}_{2}$ & Ordem \\
\hline Avançado & 0,225 & 4 & 0,067 & 5 \\
Intermediário & $-0,173$ & 10 & 0,114 & 2 \\
Atrasado & $-0,277$ & 12 & $-0,227$ & 14 \\
Janeiro & 0,269 & 2 & $-0,045$ & 10 \\
Fevereiro & 0,304 & 1 & $-0,186$ & 13 \\
Março & 0,248 & 3 & 0,033 & 8 \\
Abril & $-0,138$ & 8 & 0,057 & 6 \\
Maio & $-0,347$ & 13 & $-0,316$ & 15 \\
Junho & $-0,482$ & 15 & $-0,064$ & 12 \\
Julho & $-0,450$ & 14 & 0,012 & 9 \\
Agosto & $-0,173$ & 9 & 0,101 & 3 \\
Setembro & $-0,265$ & 11 & 0,292 & 1 \\
Outubro & $-0,126$ & 7 & 0,092 & 4 \\
Novembro & 0,001 & 5 & 0,046 & 7 \\
Dezembro & $-0,005$ & 6 & $-0,057$ & 11 \\
VI (1) (\%) & 72,6 & & 20,3 & \\
VA ${ }^{(2)}$ (\%) & 72,6 & & 92,9 & \\
\hline
\end{tabular}

(1) Variação individual. ${ }^{(2)}$ Variação acumulada.

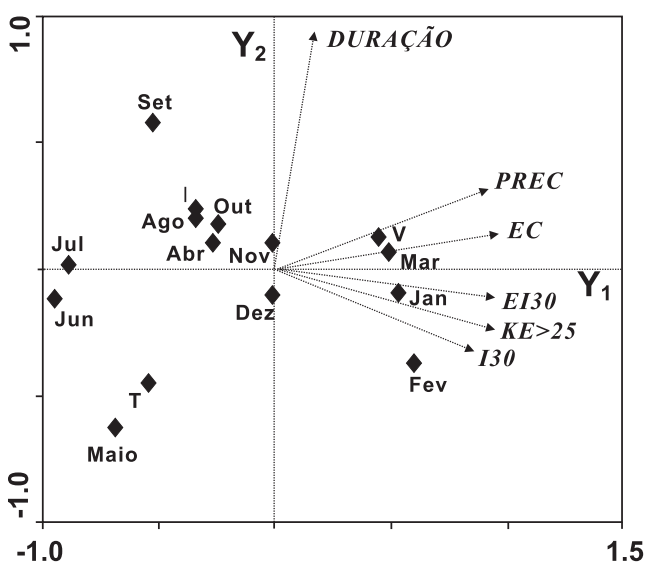

Figura 2. Análise de componentes principais para os dados de precipitação e erosividade das chuvas. V; I e T: padrões avançado, intermediário e atrasado, respectivamente.

separação dos meses e padrões foram: energia cinética (Ec), $\mathrm{EI}_{30}, \mathrm{KE}>25$, altura precipitada (PREC) e $\mathrm{I}_{30}$, pois apresentaram coeficientes de correlação $(\mathrm{rXiYj})$ superiores a 0,85 com $\mathrm{Y}_{1}$ (Quadro 7). A duração das chuvas pouco influenciou na distribuição dos meses e padrões em relação ao primeiro componente.

Pode-se concluir que os meses de junho, julho e maio e o padrão atrasado, com menores valores de $\mathrm{Y}_{1}$, apresentaram valores inferiores à média geral para PREC, EC, $\mathrm{EI}_{30}$ e $\mathrm{I}_{30}$ e $\mathrm{KE}>25$, e os meses de janeiro, fevereiro e março e o padrão avançado, com os maiores $\mathrm{Y}_{1}$, apresentaram valores, para estas variáveis, acima da média geral (Quadro 6 e Figura 2). Nos demais meses e para o padrão de precipitação intermediário, os valores encontrados dessas variáveis ficaram próximos à média geral, caracterizando o período de transição entre as estações seca e chuvosa.

Analisando o eixo $\mathrm{Y}_{2}$, observa-se que a única variável que apresentou correlação alta com esse eixo foi a duração da chuva (rXiYj=0,96) (Quadro 7). Em relação aos meses do ano e padrões de precipitação, verifica-se que a maioria ficou em torno da média geral. Entretanto, destaca-se o mês de setembro, que apresentou durações de chuvas superiores à média geral, e o mês de maio e o padrão atrasado, com menores valores desse atributo.

Esses resultados complementam aqueles encontrados na análise univariada, caracterizando de forma clara o período seco (maio-julho) e o período chuvoso (janeiro a março). É possível constatar que mais do que a altura precipitada $(\mathrm{rXiYj}=0,92)$, as variáveis $\mathrm{Ec}, \mathrm{EI}_{30}$ e $\mathrm{KE}>25$ caracterizam de maneira decisiva o potencial erosivo das chuvas no período de janeiro a março, destacando a ocorrência de maior número de chuvas de elevada energia cinética (Figura 2 e Quadro 7). Em complemento, neste mesmo período, as chuvas apresentam-se com pequena duração $(r X i Y j=0,18)$, confirmando a ocorrência de 
Quadro 7. Coeficientes de correlação linear (rXiYj) entre as variáveis originais (Xi) e os dois primeiros componentes principais ( $\mathrm{Y} 1$, e $\mathrm{Y} 2)$ para os dados dos atributos físicos das chuvas $\mathrm{e}$ erosividade

\begin{tabular}{lcc}
\hline Variável original $\left(\mathbf{X}_{\mathbf{i}}\right)$ & $\mathbf{Y}_{\mathbf{1}}$ & $\mathbf{Y}_{\mathbf{2}}$ \\
\hline Precipitação pluviométrica & 0,92 & 0,32 \\
Duração das chuvas & 0,18 & 0,96 \\
Energia cinética & 0,97 & 0,14 \\
I30 & 0,86 & $-0,32$ \\
EI 30 & 0,95 & $-0,12$ \\
KE $>25$ & 0,94 & $-0,23$ \\
\hline
\end{tabular}

eventos convectivos que se caracterizam por grande intensidade $(r X i Y j=0,86)$ e curta duração, resultando em maior poder erosivo.

A região de estudo possui um relevo bastante acidentado, denominado "mar de morros' (Ab'Saber, 1970). Na parte não ocupada por floresta (Estação Ecológica de Piraí) ou fragmentos da mata Atlântica, assim como todo o Médio Vale do rio Paraíba do Sul, o solo é utilizado principalmente com a pecuária extensiva, caracterizada por baixa cobertura vegetal proporcionada pelo superpastejo, queimadas freqüentes e preparo de solo morro abaixo em decorrência da formação ou reforma das pastagens, sendo encontradas diferentes formas erosivas. Portanto, com base na distribuição da erosividade, uma das formas de reduzir os problemas associados à erosão hídrica nessa região está relacionado à mudança do tipo de manejo e uso de práticas conservacionistas, principalmente no período de novembro a março, quando as chuvas apresentam o seu maior potencial erosivo.

\section{CONCLUSÕES}

1. A erosividade anual de Ribeirão das Lajes- RJ está concentrada no período de novembro a março, com $81,3 \%$ do total anual, e é classificada de média a forte, sendo de $6.772 \mathrm{MJ} \mathrm{mm} \mathrm{ha}^{-1} \mathrm{~h}^{-1} \mathrm{o}$ fator $\mathrm{R}$ local.

2. O padrão de precipitação pluvial predominante na região de estudo é o avançado (45,7\%), seguido do padrão atrasado (30,8 \%) e intermediário (23,6 \%).

3. De acordo com a técnica de ACP, as características das chuvas e índices de erosividade que estiveram mais correlacionadas com os meses e padrões de precipitação foram: energia cinética, $\mathrm{EI}_{30}$, $\mathrm{KE}>25$, altura precipitada e $\mathrm{I}_{30}$; sendo o padrão avançado associado ao período chuvoso (janeiro a março) e o atrasado aos meses mais secos (maio a julho).

\section{LITERATURA CITADA}

AB'SABER, A.N. Províncias geológicas e domínios morfoclimáticos no Brasil. Geomorfologia, 20:1-26, 1970.

ALBUQUERQUE, A.W.; MOURA FILHO, G.; SANTOS, J.R.; COSTA, J.P.V. \& SOUZA, J.L. Determinação de fatores da equação universal de perda de solo nas condições de Sumé, PB. R. Bras. Eng. Agríc. Amb., 9:180-188, 2005.

BERTOL, I.; LEITE, D.; GUADAGNIN, J.C. \& RITTER, S.R. Erosão hídrica em um Nitossolo Háplico submetido a diferentes sistemas de manejo sob chuva simulada: II. Perdas de nutrientes e carbono orgânico. R. Bras. Ci. Solo, 28:1045-1054, 2004.

BOUROCHE, J.M. \& SAPORTA, G. Análise de dados. Rio de Janeiro, Zahar, 1982. 117p.

CABEDA, M.S.V. Computation of storm EI values. West Lafayette, Purdue University 1976. 6p. (Não Publicado)

CARVALHO, D.F.; MONTEBELLER, C.A.; FRANCO, E.M.; VALCARCEL, R. \& BERTOL, I. Padrões de precipitação e índices de erosividade para as chuvas de Seropédica e Nova Friburgo-RJ. R. Bras. Eng. Agríc. Amb., 9:7-14, 2005.

CARVALHO, D.F.; CRUZ, E.S.; PINTO, M.F.; SILVA, L.D.B. \& GUERRA, J.G.M. Características da chuva e perdas por erosão para diferentes práticas de manejo do solo. R. Bras. Eng. Agríc. Amb., 2008. (aceito para publicação)

CARVALHO, N.O. Hidrossedimentologia prática. Rio de Janeiro, CPRM - Companhia de Pesquisa em Recursos Minerais, 1994. 372p.

COMITÊ PARA INTEGRAÇÃO DA BACIA HIDROGRÁFICA DO RIO PARAÍBA DO SUL - CEIVAP. Diagnóstico da situação atual dos recursos hídricos. Rio de Janeiro, Fundação Coppetec/UFRJ/ANA, 2002. 1v.

COGO, C.M.; ELTZ, F.L.F.; CASSOL. A. \& SANTOS, M.V Erosividade das chuvas de Uruguaiana-RS, determinada pelo índice $\mathrm{EI}_{30}$, no período de 1963-1990. In: CONGRESSO BRASILEIRO DE CIÊNCIA DO SOLO, 29., Ribeirão Preto, 2003. Anais. Ribeirão Preto, Sociedade Brasileira de Ciência do Solo, 2003. CD-ROM.

ELTZ, F.L.F.; MEHL, H.U. \& REICHERT, J.M. Perdas de solo e água em entressulcos em um Argissolo VermelhoAmarelo submetido a quatro padrões de chuvas. R. Bras. Ci. Solo, 25:485-493, 2001.

EVANGELISTA, A.W.P.; CARVALHO, L.G. \& BERNARDINO, D.T. Caracterização do padrão das chuvas ocorrentes em Lavras, MG. Irriga, 10:306-317, 2005.

FERREIRA, D.F. Sistema para análise de variância para dados balanceados (SISVAR versão 4.3). Lavras, Universidade Federal de Lavras, 2003.

FLANAGAN, D.C.; FOSTER, G.R. \& MOLDENHAUER, W.C. Storm pattern effect on infiltration, runoff and erosion. Trans. Am. Soc. Agron. Eng., 31:414-420, 1988. 
FOSTER, G.R.; McCOOL, D.K.; RENARD, K.G. \& MOLDENHAUER, W.C. Conversion of the universal soil loss equation to SI metric units. J. Soil Water Conserv., 36:355-359, 1981.

GONÇALVES, F.A. Erosividade das chuvas no Estado do Rio de Janeiro. Viçosa, MG, Universidade Federal de Viçosa, 2002. 120p. (Tese de Mestrado)

GONÇALVES, F.A.; SILVA, D.D.; PRUSKI, F.F.; CARVALHO, D.F. \& CRUZ, E.S. Índices e espacialização da erosividade das chuvas para o Estado do Rio de Janeiro. R. Bras. Eng. Agríc. Amb., 10:269-276, 2006.

HORNER, W.W. \& JENS, S.W. Surface runoff determination from rainfall without using coefficients. Trans. Am. Soc. Agron. Eng., 107:1039-1117, 1941.

LIGHT. Lajes: 20 anos da epopéia. 1997. 40p.

MEHL, H.U.; ELTZ, F.L.F.; REICHERT, J.M. \& DIDONÉ, I.A. Caracterização de padrões de chuvas ocorrentes em Santa Maria (RS). R. Bras. Ci. Solo, 25:475-483, 2001.

MONTEBELLER, C.A. Variabilidade espacial do potencial erosivo das chuvas no Estado do Rio de Janeiro. Seropédica, Universidade Federal Rural do Rio de Janeiro, 2005. 103p. (Tese de Mestrado)

MONTEBELLER, C.A.; CEDDIA, M.B.; CARVALHO, D.F.; VIEIRA, S.R. \& FRANCO, E.M. Variabilidade espacial do potencial erosivo das chuvas no Estado do Rio de Janeiro. Eng. Agríc., 27:426-435, 2007.

MORETI, D.; CARVAlHo, M.P.; MANNIGEL, A.R. \& MEDEIROS, L.R. Importantes características de chuva para a conservação do solo e da água no município de São Manuel (SP). R. Bras. Ci. Solo, 27:713-725, 2003.

MOURA, A.R.B. \& MEDEIROS, J.F. Determinação inicial da erosividade da chuva (fator R) em 1985, em Mossoró (RN). R. Bras. Ci. Solo, 11:229-231, 1987.

OLIVEIRA JUNIOR, R.C. \& MEDINA, B.F. A erosividade das chuvas em Manaus (AM). R. Bras. Ci. Solo, 14:235-239, 1990.
PEÑALVA BAZZANO, M.G.; ELTZ, F.L.F. \& CASSOL, E.A. Erosividade, coeficiente de chuva, padrões e período de retorno das chuvas de Quarai, RS. R. Bras. Ci. Solo, 31:1205-1217, 2007.

SHEN, H.W. \& LAI, J.S. Sustain reservoir useful life by flushing sediment. Int. J. Sediment Res., 11:11-17, 1996.

SILVA, A.B.; RESENDE, M.; SOUSA, A.R. \& MARGOLIS, E. Mobilização do solo, erosão e produtividade de milho e feijão em um Regossolo no Agreste de Pernambuco. Pesq. Agropec. Bras., 34:299-307, 1999.

SILVA, M.L.N.; FREITAS, P.L.; BLANCANEAUX, P.; CURI, N. \& LIMA, J.M. Relação entre parâmetros da chuva e perdas de solo e determinação da erodibilidade de um Latossolo Vermelho-Escuro em Goiânia (GO). R. Bras. Ci. Solo, 21:131-137, 1997a.

SILVA, M.L.N.; FREITAS, P.L.; BLANCANEAUX, P. \& CURI, N. Índices de erosividade das chuvas da região de Goiânia, GO. Pesq. Agropec. Bras., 32:977-985, 1997b.

TÁVORA, M.R.P.; SILVA, J.C.R.; HERNÁNDES, F.F.F.; SAUNDERS, L.C.U. \& MOREIRA, E.G.S. Perdas de solo, água e nutrientes em Latossolo Vermelho-Amarelo distrófico de Ubajara (CE). R. Bras. Ci. Solo, 9:63-66, 1985.

TER BRAAK, C.J.F. \& SMILAUER, P. Canoco-reference manual and user's guide to canoco for windows: Software for canonical Community Ordination (Version 4). Microcomputer Power. Ithaca, 1998. 332p.

WISCHMEIER, W.H. A rainfall erosion index for a universal soil loss equation. Soil Sci. Soc. Am. Proc., 23:246-249, 1959.

WISCHMEIER, W.H. \& SMITH, D.D. Predicting rainfallerosion losses from cropland East of the Rocky Mountains: Guide for selection of practices for soil and water conservation. Washington, USDA, 1965. 47p.

WISCHMEIER, W.H. \& SMITH, D.D. Rainfall energy and its relationships to soil loss. Trans. Am. Geophys. Union, 39:285-291, 1958. 